\title{
The study of heat transfer phenomena using PM for approximate solution with Dirichlet and mixed boundary conditions
}

\author{
U. Filobello-Nino ${ }^{1}$, H. Vazquez-Leal ${ }^{1, ~}{ }^{*}$, A. Sarmiento-Reyes ${ }^{2}$, A. Perez-Sesma ${ }^{1}$, \\ L. Hernandez-Martinez ${ }^{2}$, A. Herrera-May ${ }^{3}$, V. M. Jimenez-Fernandez ${ }^{1}$, A. Marin-Hernandez, \\ D. Pereyra-Diaz, A. Diaz-Sanchez ${ }^{2}$
}

\begin{abstract}
${ }^{1}$ Electronic Instrumentation and Atmospheric Sciences School, Universidad Veracruzana, Circuito Gonzalo Aguirre Beltrán S/N, Xalapa, Veracruz, Mexico 91000

${ }^{2}$ National Institute for Astrophysics, Optics and Electronics, Luis Enrique Erro \#1, Sta. María Tonantzintla. Puebla, México, 72840

${ }^{3}$ Micro and Nanotechnology Research Center, Universidad Veracruzana, Calzada, Ruiz Cortines, Boca del Rio 94292, Veracruz, Mexico

${ }^{4}$ Department of Artificial Intelligence, Universidad Veracruzana, Sebastián Camacho No. 5, C.P. 91000 Xalapa, Veracruz, Mexico
\end{abstract}

\section{Email address:}

hvazquez@uv.mx (H. Vazquez-Leal)

\section{To cite this article:}

U. Filobello-Nino, H. Vazquez-Leal, A. Sarmiento-Reyes, A. Perez-Sesma, L. Hernandez-Martinez, A. Herrera-May, V. M. Jimenez-Fernandez, A. Marin-Hernandez, D. Pereyra-Diaz1, A. Diaz-Sanchez. The Study of Heat Transfer Phenomena Using PM for Approximate Solution with Dirichlet and Mixed Boundary Conditions. Applied and Computational Mathematics. Vol. 2, No. 6, 2013, pp. 143-148. doi: 10.11648/j.acm.20130206.16

\begin{abstract}
In this paper, we present Perturbation Method (PM) to solve nonlinear problems. As case study PM is employed to obtain approximate solutions for differential equations related with heat transfer phenomena. Comparing figures between approximate and exact solutions, show the effectiveness of the method.
\end{abstract}

Keywords: Dirichlet Boundary conditions, Mixed Boundary Conditions, Nonlinear Differential Equation, Perturbation Method, Approximate Solutions

\section{Introduction}

The heat transfer laws are of paramount importance in the design and operation of equipment in many industrial applications as well as in pure sciences. Therefore it is important to search for accurate approximate solutions to the equations describing these phenomena. However, it is well known that nonlinear differential equations that describe them, are difficult to solve.

The perturbation method (PM) is a well established method; it is among the pioneer techniques to approach various kinds of nonlinear problems. This procedure was originated by S. D. Poisson and extended by J. H. Poincare. Although the method appeared in the early $19^{\text {th }}$ century, the application of a perturbation procedure to solve nonlinear differential equations was performed later on that century. The most significant efforts were focused on celestial mechanics, fluid mechanics, and aerodynamics [2, 37].

In a broad sense, it is possible to express a nonlinear differential equation in terms of one linear part and other nonlinear. The nonlinear part is considered as a small perturbation through a small parameter (the perturbation parameter). The assumption that the nonlinear part is small compared to the linear is considered as a disadvantage of the method. There are other modern alternatives to find approximate solutions to the differential equations that describe some nonlinear problems such as those based on: variational approaches [5-7, 29], tanh method [8], exp-function [9, 10], Adomian's decomposition method [11-16], parameter expansion [17], homotopy perturbation method [3,4,16,18-29,31-36,38,42], homotopy analysis method [30], Homotopy asymptotic method [1], and perturbation method [39,41] among many others. Also, a few exact solutions to nonlinear differential equations have been reported occasionally [40].

Although the PM method provides in general, better results for small perturbation parameters $\mathcal{E}<<1$, we will see that our approximations, besides to be handy, have a good accuracy, even for relatively large values of the perturbation parameter [39,41].

This paper is organized as follows. Section 2 presents a 
survey about the works related with this paper. In Section 3, we introduce the basic idea of the PM method. For Section 4, we provide an application of PM method, by solving a couple of examples with applications in sciences and engineering. Section 5 discusses the main results obtained. Finally, a brief conclusion is given in Section 6.

\section{Survey of the Related Works}

Given the importance of phenomena involving heat flow, other sophisticated methods have been used to find approximate solutions to these problems, such as Homotopy Perturbation Method (HPM) [16,43,44], in [16] is compared a modified version of HPM and Adomian decomposition method in order to evaluate the temperature distribution of a straight rectangular fin with temperature dependent surface heat flux for all possible types of heat transfer, in [43] HPM is employed to solve for the temperature distribution in lumped system of combined convection radiation, in [44] HPM and PM are used to find analytical approximate solutions, for an unsteady nonlinear convective-radiative equation and a nonlinear convective-radiative conduction equation containing two small parameters. Laplace Transform Homotopy Perturbation Method (LT-HPM) [42], [42] employs LT-HPM, to approximately solve the differential equation which describes the temperature distribution in a uniformly thick rectangular fin radiation to free space with nonlinearity of high order (see below, this study proposes an approximate solution for this equation), Homotopy Asymptotic Method [1] (where is analyzed the cooling of a system with variable specific heat, also is analysed the temperature distribution in a uniformly thick rectangular fin radiation to free space with nonlinearity of high order), Adomian's decomposition method [16], among others.

\section{Basic Idea of Perturbation Method}

Let the differential equation of one dimensional nonlinear system be in the form

$$
L(x)+\varepsilon N(x)=0,
$$

where we assume that $x$ is a function of one variable $x=x(t), L(x)$ is a linear operator which, in general, contains derivatives in terms of $t, N(x)$ is a nonlinear operator, and $\mathcal{E}$ is a small parameter.

Considering the nonlinear term in (1) to be a small perturbation and assuming that the solution for (1) can be written as a power series in the small parameter $\mathcal{E}$,

$$
x(t)=x_{0}(t)+\varepsilon x_{1}(t)+\varepsilon^{2} x_{2}(t)+\ldots
$$

Substituting (2) into (1) and equating terms having identical powers of $\mathcal{E}$, we obtain a number of differential equations that can be integrated, recursively, to find the values for the functions: $x_{0}(t), x_{1}(t), x_{2}(t)$

\section{Approximate Solution of Equations}

\subsection{Case study 1}

The following example, emphasizes the use of HPM for solving nonlinear ODEs with Dirichlet boundary conditions.

We will consider the following nonlinear differential equation, which describes the cooling of a system with variable specific heat [1].

$$
\frac{d^{2} y(x)}{d x^{2}}+\varepsilon\left[y(x) \frac{d^{2} y(x)}{d x^{2}}+\left(\frac{d y(x)}{d x}\right)^{2}\right]=0,0 \leq x \leq 1, y(0)=1, y(1)=0
$$

where $\mathcal{E}$ is a positive parameter.

It is possible to find a handy solution for (3) by applying the PM method.

Identifying terms:

$$
\begin{aligned}
& L(y)=y^{\prime \prime}(x), \\
& y(x)=y_{0}(x)+\varepsilon y_{1}(x)+\mathcal{E}^{2} y_{2}(x)+\mathcal{E}^{3} y_{3}(x)+\mathcal{E}^{4} y_{4}(x)+\ldots, \text { see (2)). }
\end{aligned}
$$

$$
N(y)=y(x) y^{\prime \prime}(x)+y^{\prime 2}(x)
$$

where prime denotes differentiation respect to $\mathrm{x}$.

Identifying $\varepsilon$ with the PM parameter, we assume a

On comparing the coefficients of like powers of $\mathcal{E}$ it can be solved for $y_{0}(x), y_{1}(x), y_{2}(x), y_{3}(x), \ldots$, and so on. Later it will be seen that, a very good handy result is obtained, by keeping up to fifth order approximation.

$$
\begin{gathered}
\left.\varepsilon^{0}\right) y_{0}^{\prime \prime}=0, \quad y_{0}(0)=1, \quad y_{0}(1)=0, \\
\left.\varepsilon^{1}\right) y_{1}^{\prime \prime}+y_{0}^{\prime 2}+y_{0} y_{0}^{\prime \prime}=0, y_{1}(0)=0, \quad y_{1}(1)=0, \\
\left.\varepsilon^{2}\right) y_{2}^{\prime \prime}+y_{1} y_{0}^{\prime \prime}+y_{0} y_{1}^{\prime \prime}+2 y_{1}^{\prime} y_{0}^{\prime}=0, \quad y_{2}(0)=0, \quad y_{2}(1)=0,
\end{gathered}
$$




$$
\begin{gathered}
\left.\varepsilon^{3}\right) y_{3}^{\prime \prime}+y_{2} y_{0}^{\prime \prime}+y_{0} y_{2}^{\prime \prime}+y_{1} y_{1}^{\prime \prime}+y_{1}^{\prime 2}+2 y_{2}^{\prime} y_{0}^{\prime}=0 \quad y_{3}(0)=0 \quad y_{3}(1)=0 \\
\left.\mathcal{E}^{4}\right) y_{4}^{\prime \prime}+y_{3} y_{0}^{\prime \prime}+y_{1} y_{2}^{\prime \prime}+y_{0} y_{3}^{\prime \prime}+y_{2} y_{1}^{\prime \prime}+2 y_{3}^{\prime} y_{0}^{\prime}+2 y_{1}^{\prime} y_{2}^{\prime}, y_{4}(0)=0, y_{4}(1)=0 \\
\left.\mathcal{E}^{5}\right) \begin{array}{c}
y_{5}^{\prime \prime}+y_{0} y_{4}^{\prime \prime}+\left(y_{2}^{\prime}\right)^{2}+2 y_{0}^{\prime} y_{4}^{\prime}+y_{1} y_{3}^{\prime \prime}, y_{5}(0)=0, \quad y_{5}(1)=0 \\
+y_{2} y_{2}^{\prime \prime}+2 y_{1}^{\prime} y_{3}^{\prime}+y_{3} y_{1}^{\prime \prime}+y_{4} y_{0}^{\prime \prime}=0
\end{array}
\end{gathered}
$$

Thus, the results obtained are

$$
\begin{gathered}
y_{0}(x)=-x+1, \\
y_{1}(x)=-\frac{1}{2} x^{2}+\frac{1}{2} x, \\
y_{2}(x)=-\frac{1}{2} x^{3}+x^{2}-\frac{1}{2} x, \\
y_{3}(x)=-\frac{5}{8} x^{4}+\frac{7}{4} x^{3}-\frac{13}{8} x^{2}+\frac{1}{2} x, \\
y_{4}(x)=-\frac{7}{8} x^{5}+\frac{25}{8} x^{4}-\frac{33}{8} x^{3}+\frac{19}{8} x^{2}-\frac{1}{2} x, \\
y_{5}(x)=-\frac{21}{16} x^{6}+\frac{91}{16} x^{5}-\frac{155}{16} x^{4}+\frac{129}{16} x^{3}-\frac{13}{4} x^{2}+\frac{1}{2} x .
\end{gathered}
$$

By substituting (13)-(18) into (6) we obtain a fifth order approximation for the solution of (3).

We consider as a case study, the value of parameter $\varepsilon=1$; to obtain a handy approximate solution

$$
\begin{gathered}
y(x)=-\frac{1}{2} x-2 x^{2}+\frac{83}{16} x^{3}-\frac{115}{16} x^{4}+\frac{77}{16} x^{5}-\frac{21}{16} x^{6}+1 \\
\left.y(x)=y_{0}(x)+\varepsilon y_{1}(x)+\mathcal{E}^{2} y_{2}(x)+\mathcal{E}^{3} y_{3}(x)+\mathcal{E}^{4} y_{4}(x)+\ldots, \text { see }(2)\right)
\end{gathered}
$$

As a matter of fact, we will see that (19) is also, highly accurate.

\subsection{Case Study 2}

The following example, emphasizes the use of HPM for solving nonlinear ODEs with mixed boundary conditions.

We will consider the following nonlinear differential equation, which describes the temperature distribution in a uniformly thick rectangular fin radiation to free space with nonlinearity of high order [1].

$$
\frac{d^{2} y(x)}{d x^{2}}-\varepsilon y(x)^{4}=0,0 \leq x \leq 1, y^{\prime}(0)=0, y(1)=1,
$$

where $\mathcal{E}$ is a positive parameter.

To apply PM method, we identify terms as follows

$$
\begin{gathered}
L(y)=y^{\prime \prime}(x), \\
N(y)=-y(x)^{4},
\end{gathered}
$$

where prime denotes differentiation respect to $\mathrm{x}$.

Identifying $\mathcal{E}$ with the PM parameter, we assume a

Equating the terms with identical powers of $\mathcal{E}$ it can be solved for $y_{0}(x), y_{1}(x), y_{2}(x), \ldots$, and so on. We will see that a handy result is obtained, by keeping up to fifth order approximation.

$$
\begin{gathered}
\left.\mathcal{E}^{0}\right) y_{0}^{\prime \prime}=0, \quad y_{0}^{\prime}(0)=0, \quad y_{0}(1)=1, \\
\left.\mathcal{E}^{1}\right) y_{1}^{\prime \prime}-y_{0}{ }^{4}=0, \quad y_{1}^{\prime}(0)=0, \quad y_{1}(1)=0, \\
\left.\mathcal{E}^{2}\right) y_{2}^{\prime \prime}-4 y_{0}^{3} y_{1}=0, \quad y_{2}^{\prime}(0)=0, \quad y_{2}(1)=0, \\
\left.\mathcal{E}^{3}\right) y_{3}^{\prime \prime}-4 y_{0}{ }^{2} y_{1}{ }^{2}-2 y_{0}{ }^{2}\left(y_{1}{ }^{2}+2 y_{0} y_{2}\right)=0, y_{3}^{\prime}(0)=0, \quad y_{3}(1)=0, \\
\left.\mathcal{E}^{4}\right) y_{4}^{\prime \prime}-2 y_{0}^{2}\left(2 y_{3} y_{0}+2 y_{1} y_{2}\right)-4\left(y_{1}{ }^{2}+2 y_{2} y_{0}\right) y_{0} y_{1}=0, y_{4}^{\prime}(0)=0, \quad y_{4}(1)=0, \\
\left.\varepsilon^{5}\right) \begin{array}{l}
y_{5}^{\prime \prime}-2 y_{0}{ }^{2}\left(y_{2}{ }^{2}+2 y_{0} y_{4}+2 y_{1} y_{3}\right) \\
-4 y_{0} y_{1}\left(2 y_{0} y_{3}+2 y_{1} y_{2}\right)-\left(y_{1}{ }^{2}+2 y_{0} y_{2}\right)^{2}=0 \quad, y_{5}^{\prime}(0)=0, \quad y_{5}(1)=0,
\end{array}
\end{gathered}
$$

After solving the above equations, one obtains

$$
y_{0}(x)=1 \text {, }
$$

$$
y_{1}(x)=\frac{1}{2} x^{2}-\frac{1}{2}
$$




$$
\begin{gathered}
y_{2}(x)=\frac{1}{6} x^{4}-x^{2}+\frac{5}{6}, \\
y_{3}(x)=\frac{13}{180} x^{6}-\frac{7}{12} x^{4}+\frac{29}{12} x^{2}-\frac{343}{180}, \\
y_{4}(x)=\frac{23}{720} x^{8}-\frac{13}{36} x^{6}+\frac{133}{72} x^{4}-\frac{1181}{180} x^{2}+\frac{3631}{720}, \\
y_{5}(x)=\frac{929}{64800} x^{10}-\frac{299}{1440} x^{8}+\frac{611}{432} x^{6}-\frac{12677}{2160} x^{4}+\frac{27601}{1440} x^{2}-\frac{940859}{64800} .
\end{gathered}
$$

By substituting (30)-(35) into (23) we obtain a fifth order approximation for the solution of (20).

Considering as a case study, the value of parameter $\varepsilon=0.30$; we obtain a handy approximate solution

$$
\begin{aligned}
y(x)= & \frac{70329323}{80000000}+\frac{1898907}{16000000} x^{2}-\frac{393}{8000000} x^{4} \\
& +\frac{3939}{1600000} x^{6}-\frac{3933}{16000000} x^{8}+\frac{2787}{80000000} x^{10} .
\end{aligned}
$$

We will see that (36) has also, a good precision.

\section{Discussion}

The fact that the PM depends on a parameter which is assumed small, suggests that the method is for a limited use. In this work, PM method has been applied, successfully to the problem of finding approximate solutions for nonlinear differential equations with Dirichlet and mixed boundary conditions, that describe some heat transfer phenomena. Although the solutions reported for other sophisticated methods (see section 2) have good accuracy, they are more complicated for applications than PM.

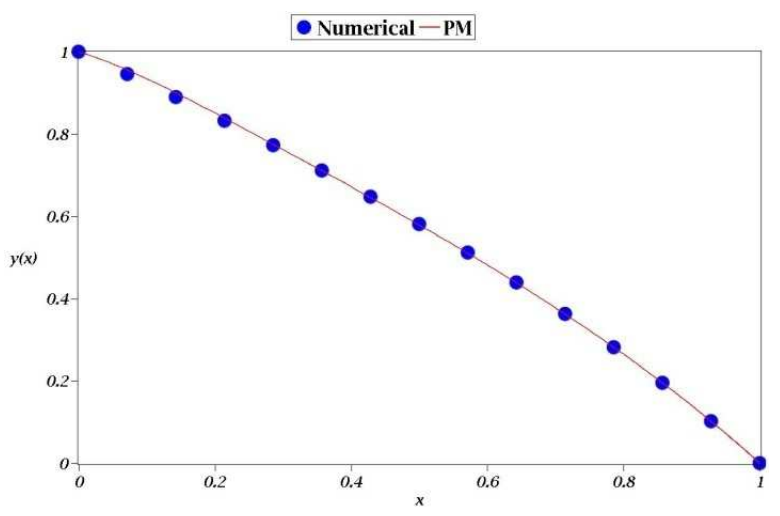

Fig 1. Approximation of (3) and RK4 comparison for $\varepsilon=1$

Fig.1 shows the comparison between approximate solution (19) for differential equation with Dirichlet boundary conditions (3), with the four order Runge Kutta (RK4) numerical solution. It can be noticed that figures are in good agreement showing the accuracy of (19). This proves the efficiency of PM method in this case, despite of the fact that it was only considered the fifth-order approximation.

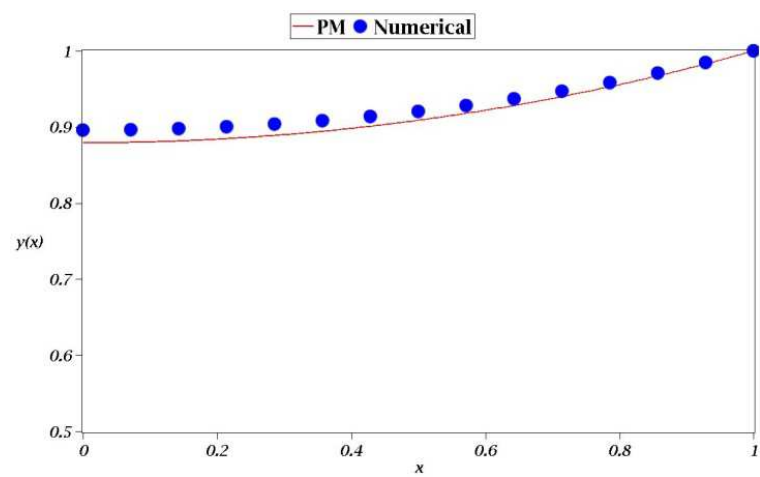

Fig 2. Approximation of (20) and RK4 comparison for $\varepsilon=0.30$

On the other hand, Fig.2 compares approximate solution (36) for (20) with the four order Runge Kutta (RK4) numerical solution. From figures it is deduced that (36) has an acceptable precision, taking into account that problems with mixed boundary conditions, have the added difficulty of not provide one of the endpoints of the interval, in this case $y(0)$. It is noted that (36) is only the fifth-order approximation to the equation to be solved. Therefore, accuracy can be increased, considering higher order approximations.

It is well known that PM method provides in general, better results for small perturbation parameters $\mathcal{E}<<1$ (see (1)) and when are included the most number of terms from (2). To be precise, $\mathcal{E}$ is a parameter of smallness, that measure how greater is the contribution of linear term $L(x)$ than the one of $N(x)$ in (1). Fig. 1 and Fig. 2 show a noticeable fact, that (19) and (36) turn out to be a good approximation of (3) and (20) respectively, although perturbation parameters $\varepsilon=1$ and $\varepsilon=0.30$ cannot be considered small.

Finally, our approximate solutions (19) and (36) do not depend of any adjustment parameter, for which, are in principle, general expressions for proposed problems.

\section{Conclusions}

In this study, PM was presented to construct analytical approximate solutions for nonlinear differential equations in the form of rapidly convergent series. In order to prove the versatility of this method, we proposed as examples a couple of equations governed by Dirichlet and mixed boundary conditions, with good results. The success of PM for this case, it has to be considered as a possibility to apply it in other non linear problems, instead of using other sophisticated and difficult methods. From Fig. 1 and Fig. 2, it is deduced that the proposed solutions have good precision.

\section{References}

[1] Vasile Marinca and Nicolae Herisanu, Nonlinear Dynamical Systems in Engineering, first edition, Springer-Verlag Berlin Heidelberg, 2011. 
[2] Chow, T.L., Classical Mechanics. John Wiley and Sons Inc., USA. 1995.

[3] He, J.H., A coupling method of a homotopy technique and a perturbation technique for nonlinear problems. Int. J. Non-Linear Mech., 351 (1998) 37-43. DOI: 10.1016/S0020-7462(98)00085-7

[4] He, J.H.,. Homotopy perturbation technique, Comput. Methods Applied Mech. Eng., 178 (1999) 257-262. DOI: 10.1016/S0045-7825(99)00018-3

[5] Assas, L.M.B., Approximate solutions for the generalized K-dV- Burgers' equation by He's variational iteration method. Phys. Scr., $76 \quad$ (2007) 161-164. DOI: $10.1088 / 0031-8949 / 76 / 2 / 008$

[6] He, J.H., Variational approach for nonlinear oscillators. Chaos, Solitons and Fractals, 34 (2007) 1430-1439. DOI: 10.1016/j.chaos.2006.10.026

[7] Kazemnia, M., S.A. Zahedi, M. Vaezi and N. Tolou, Assessment of modified variational iteration method in BVPs high-order differential equations. Journal of Applied $\begin{array}{llll}\text { Sciences, } & 8 & \text { (2008) }\end{array}$ DOI:10.3923/jas.2008.4192.4197

[8] Evans, D.J. and K.R. Raslan, The Tanh function method for solving some important nonlinear partial differential. Int. J. Computat. Math., 82 (2005) 897-905. DOI: $10.1080 / 00207160412331336026$

[9] $\mathrm{Xu}, \mathrm{F}$., A generalized soliton solution of the Konopelchenko-Dubrovsky equation using exp-function method. ZeitschriftNaturforschung - Section A Journal of Physical Sciences, 62(12) (2007) 685-688.

[10] Mahmoudi, J., N. Tolou, I. Khatami, A. Barari and D.D. Ganji, Explicit solution of nonlinear ZK-BBM wave equation using Exp-function method. Journal of Applied Sciences, 8 (2008) 358-363. DOI:10.3923/jas.2008.358.363

[11] Adomian, G., A review of decomposition method in applied mathematics. Applications. 135 (1988) 501-544.

[12] Babolian, E. and J. Biazar, On the order of convergence of Adomian method. Applied Mathematics and Computation, 130(2) (2002) 383-387. 10.1016/S0096-3003(01)00103-5

[13] Kooch, A. and M. Abadyan, Efficiency of modified Adomian decomposition for simulating the instability of nano-electromechanical switches: comparison with the conventional decomposition method. Trends in Applied Sciences Research, 7 (2012) 57-67. DOI:10.3923/tasr.2012.57.67

[14] Kooch, A. and M. Abadyan, Evaluating the ability of modified Adomian decomposition method to simulate the instability of freestanding carbon nanotube: comparison with conventional decomposition method. Journal of Applied Sciences, $11 \quad$ (2011) 3421-3428. DOI: $10.3923 /$ jas.2011.3421.3428

[15] Vanani, S. K., S. Heidari and M. Avaji, A low-cost numerical algorithm for the solution of nonlinear delay boundary integral equations. Journal of Applied Sciences, 11 (2011) 3504-3509. DOI:10.3923/jas.2011.3504.3509

[16] Chowdhury, S. H., A comparison between the modified homotopy perturbation method and Adomian decomposition method for solving nonlinear heat transfer equations. Journal $\begin{array}{llll}\text { of Applied Sciences, } 11 & \text { (2011) 1416-1420. }\end{array}$ DOI:10.3923/jas.2011.1416.1420

[17] Zhang, L.-N. and L. Xu, Determination of the limit cycle by He's parameter expansion for oscillators in a potential. ZeitschriftfürNaturforschung - Section A Journal of Physical Sciences, 62(7-8) (2007) 396-398.

[18] He, J.H., Homotopy perturbation method for solving boundary value problems. Physics Letters A, 350(1-2) (2006) 87-88.

[19] He, J.H., Recent Development of the Homotopy Perturbation Method. Topological Methods in Nonlinear Analysis, 31(2) (2008) 205-209.

[20] Belendez, A., C. Pascual, M.L. Alvarez, D.I. Méndez, M.S. Yebra and A. Hernández, High order analytical approximate solutions to the nonlinear pendulum by He's homotopy method. PhysicaScripta, 79(1) (2009) 1-24. DOI: 10.1088/0031-8949/79/01/015009

[21] He, J.H., A coupling method of a homotopy and a perturbation technique for nonlinear problems. International Journal of Nonlinear Mechanics, 35(1) (2000) 37-43.

[22] El-Shaed, M., Application of He's homotopy perturbation method to Volterra's integro differential equation. International Journal of Nonlinear Sciences and Numerical Simulation, 6 (2005) 163-168.

[23] He, J.H., Some Asymptotic Methods for Strongly Nonlinear Equations. International Journal of Modern Physics B, 20(10) (2006) 1141-1199. DOI: 10.1142/S0217979206033796

[24] Ganji, D.D, H. Babazadeh, F Noori, M.M. Pirouz, M Janipour. An Application of Homotopy Perturbation Method for Non linear Blasius Equation to Boundary Layer Flow Over a Flat Plate, ACADEMIC World Academic Union, ISNN 1749-3889(print), 1749-3897 (online). International Journal of Nonlinear Science, 7 (4) (2009) 309-404.

[25] Ganji, D.D., H. Mirgolbabaei , Me. Miansari and Mo. Miansari , Application of homotopy perturbation method to solve linear and non-linear systems of ordinary differential equations and differential equation of order three. Journal of Applied Sciences, 8 (2008) 1256-1261. DOI:10.3923/jas.2008.1256.1261.

[26] Fereidon, A., Y. Rostamiyan, M. Akbarzade and D.D. Ganji, Application of He's homotopy perturbation method to nonlinear shock damper dynamics. Archive of Applied Mechanics, 80(6) (2010) 641-649. DOI: 10.1007/s00419-009-0334-x.

[27] Sharma, P.R. and G. Methi, Applications of homotopy perturbation method to partial differential equations. Asian Journal of Mathematics \& Statistics, 4 (2011) 140-150. DOI:10.3923/ajms.2011.140.150

[28] Aminikhah Hossein, Analytical Approximation to the Solution of Nonlinear Blasius Viscous Flow Equation by LTNHPM. International Scholarly Research Network ISRN Mathematical Analysis, (2012), 10 pages, Article ID 957473, DOI: $10.5402 / 2012 / 957473$

[29] Noorzad, R., A. Tahmasebi Poor and M. Omidvar, Variational iteration method and homotopy-perturbation method for solving Burgers equation in fluid 
dynamics.Journal of Applied Sciences, 8 (2008) 369-373. DOI:10.3923/jas.2008.369.373

[30] Patel, T., M.N. Mehta and V.H. Pradhan, The numerical solution of Burger's equation arising into the irradiation of tumour tissue in biological diffusing system by homotopy analysis method. Asian Journal of Applied Sciences, 5 (2012) 60-66. DOI:10.3923/ajaps.2012.60.66

[31] Vazquez-Leal H., U. Filobello-Niño, R. Castañeda-Sheissa, L. Hernandez Martinez and A. Sarmiento-Reyes, Modified HPMs inspired by homotopy continuation methods . Mathematical Problems in Engineering, (2012) 20 pages, Article ID 309123, DOI:10.155/2012/309123.

[32] Vazquez-Leal H., R. Castañeda-Sheissa, U. Filobello-Niño, A. Sarmiento-Reyes, and J. Sánchez-Orea, High accurate simple approximation of normal distribution related integrals. Mathematical Problems in Engineering, (2012) 22 pages, Article ID 124029, DOI: 10.1155/2012/124029.

[33] Filobello-Niño U., H. Vazquez-Leal, R. Castañeda-Sheissa, A. Yildirim, L. HernandezMartinez, D. Pereyra Díaz, A. Pérez Sesma and C. Hoyos Reyes 2012. An approximate solution of Blasius equation by using HPM method. Asian Journal of Mathematics and Statistics, Vol. 2012, 10 pages, DOI: 10.3923 /ajms.2012, ISSN 1994-5418.

[34] Biazar, J. and H. Aminikhan, Study of convergence of homotopy perturbation method for systems of partial differential equations. Computers and Mathematics with Applications, 58 (11-12) (2009) 2221-2230.

[35] Biazar, J. andH. Ghazvini, Convergence of the homotopy perturbation method for partial differential equations. Nonlinear Analysis: Real World Applications, 10 (5) (2009) 2633-2640

[36] Filobello-Niño U., H. Vazquez-Leal, D. Pereyra Díaz, A. Pérez Sesma, R. Castañeda-Sheissa, Y. Khan, A. Yildirim, L. Hernandez Martinez, and F. Rabago Bernal, HPM Applied to Solve Nonlinear Circuits: A Study Case. Applied Mathematics Sciences, 6 (87) (2012) 4331-4344.

[37] Holmes, M.H., Introduction to Perturbation Methods. Springer-Verlag, New York, 1995.

[38] D.D. Ganji, A.R. Sahouli and M. Famouri, A New modification of He's homotopy perturbation method for rapid convergence of nonlinear undamped oscillators, Journal of Applied Mathematics and Computing, 30 (2009), 181-192.

[39] Filobello-Niño U., H. Vazquez-Leal, Y. Khan, A. Yildirim, V.M. Jimenez-Fernandez, A. L Herrera May, R. Castañeda-Sheissa, and J.Cervantes Perez. Perturbation Method and Laplace-Padé Approximation to solve nonlinear problems. Miskolc Mathematical Notes, 14(1) (2013) 89-101, ISSN: $1787-2405$.

[40] Filobello-Niño U., H. Vazquez-Leal, Y. Khan, A. Perez-Sesma, A. Diaz-Sanchez, A. Herrera-May, D. Pereyra-Diaz, R. Castañeda-Sheissa, V.M. Jimenez-Fernandez, and J. Cervantes-Perez, A handy exact solution for flow due to a stretching boundary with partial slip. Revista Mexicana de Física E, 59 (2013) 51-55, ISSN $1870-3542$.

[41] Filobello-Niño U., H. Vazquez-Leal, K. Boubaker, Y. Khan, A. Perez-Sesma, A.Sarmiento Reyes, V.M. Jimenez-Fernandez, A Diaz-Sanchez, A. Herrera-May, J. Sanchez-Orea and K. Pereyra-Castro, Perturbation Method as a Powerful Tool to Solve Highly Nonlinear Problems: The Case of Gelfand,s Equation. Asian Journal of Mathematics and Statistics, (2013) 7 pages, DOI: 10.3923 /ajms.2013, ISSN 1994-5418, 2013.

[42] Filobello-Nino U., H. Vazquez-Leal, Y. Khan, A. Perez-Sesma, A. Diaz-Sanchez, V.M. Jimenez-Fernandez, A. Herrera-May, D. Pereyra-Diaz, J.M. Mendez-Perez and J. Sanchez-Orea, Laplace transform-homotopy perturbation method as a powerful tool to solve nonlinear problems with boundary conditions defined on finite intervals, Computational and Applied Mathematics, (2013) 1-16 ISSN: 0101-8205, DOI= 10.1007/s40314-013-0073-z.

[43] Rajabi A., Ganji, D.D, H.Taherian, Application of Homotopy Perturbation Method in nonlinear heat conduction and convection equations. Physic Letters A, Elsevier 360 (2007) 570-573. DOI: 10.1016/ j.physleta.2006.08.079.

[44] D.D Ganji A Rajabi., Assessment of Homotopy Perturbation and Perturbation methods in heat radiation equations. International communication in heat and mass transfer, Elsevier 360 (2006) 391-400. DOI: 10.1016/ j.icheatmasstransfer. 2005.11.001. 